\title{
BMJ Open Effectiveness of natural S-equol supplement for premenstrual symptoms: protocol of a randomised, double-blind, placebo-controlled trial
}

\author{
Takashi Takeda, ${ }^{1}$ Masami Shiina, ${ }^{1}$ Yasutaka Chiba ${ }^{2}$
}

To cite: Takeda T, Shiina M, Chiba Y. Effectiveness of natural S-equol supplement for premenstrual symptoms: protocol of a randomised, double-blind, placebocontrolled trial. BMJ Open 2018;8:e023314. doi:10.1136/ bmjopen-2018-023314

- Prepublication history for this paper is available online. To view these files, please visit the journal online (http://dx.doi. org/10.1136/bmjopen-2018023314).

Received 31 March 2018 Revised 31 May 2018

Accepted 21 June 2018

A) Check for updates

(C) Author(s) (or their employer(s)) 2018. Re-use permitted under CC BY-NC. No commercial re-use. See rights and permissions. Published by BMJ.

${ }^{1}$ Division of Women's Health, Research Institute of Traditional Asian Medicine, Kindai University, Osaka-Sayama, Japan

${ }^{2}$ Clinical Research Center, Kindai University Hospital, OsakaSayama, Japan

Correspondence to

Dr Takashi Takeda;

take@med.kindai.ac.jp

\section{ABSTRACT}

Introduction Premenstrual syndrome (PMS) comprises a range of mood, behavioural and physical symptoms, and impairs many women's quality of life. Isoflavones are expected to stabilise the natural fluctuation of the oestrogen cycle through their selective oestrogen receptor modulator-like activities that alleviate PMS symptoms. Equol, a metabolite of a soy isoflavone converted from daidzein by specific gut bacteria, has a greater bioavailability compared with other soy isoflavones. We aim to examine the effect of natural S-equol supplements on premenstrual symptoms.

Methods and analysis This study will enrol 124 women (aged 20-45years) who have PMS symptoms and are non-equol producers in a double-blind, parallel, randomised, placebo-controlled trial, in which they will receive natural S-equol supplement (equol $10 \mathrm{mg}$ a day) or placebo, orally, twice daily, for three menstrual cycles. The primary outcome measure (Daily Record of Severity of Problems total score) will be assessed during intervention cycles. To compare the primary outcomes between the S-equol group and the placebo group, the mean differences in the Daily Record of Severity of Problems total score between the two groups will be determined. The $p$ values will be determined using Student's t-test, where the significance level is $5 \%$ (two-sided).

Ethics and dissemination The institutional review board at Kindai University approved the study. The findings of this trial will be submitted to an international peer-reviewed journal. Abstracts will be submitted to national and international conferences.

Trial registration number UMIN000031815.

\section{INTRODUCTION}

\section{Premenstrual syndrome}

Premenstrual syndrome (PMS) comprises a range of mood, behavioural and physical symptoms that are limited to the premenstrual phase. ${ }^{1}$ Epidemiological surveys have shown that the prevalence of premenstrual PMS is high $(80 \%-90 \%) .^{2}$ In approximately $5 \%$ of women, the symptoms are so severe that they interfere with personal or social relationships. ${ }^{3}$ Such a severe form of PMS is defined as premenstrual dysphoric disorder

\section{Strengths and limitations of this study}

This is the first study to assess the efficacy of equol supplement to alleviate premenstrual syndrome (PMS) symptoms.

- A key strength of this study is that we will collect data from women with PMS with strict criteria using the Daily Record of Severity of Problems.

- It remains unclear whether equol $10 \mathrm{mg}$ is a necessary and sufficient dose for alleviating PMS symptoms, and a dose finding study will be needed in the future.

(PMDD) according to the Diagnostic and Statistical Manual of Mental Disorders- $5 .{ }^{4}$ The precise pathophysiology of PMS and PMDD remains unknown, but several possible causes have been suggested, including hormonal changes, serotonergic dysfunction, stress and diet. ${ }^{5}$ Among these factors, dietary changes or supplements might be recommended as non-pharmacological medical approaches, as was concluded in a review on the treatment and management modalities for PMDD. ${ }^{6}$

\section{Isoflavones and equol}

The consumption of isoflavones, which are predominantly derived from soybeans, could reduce the risk of oestrogen-related diseases such as menopausal symptoms, breast cancer, osteoporosis and cardiovascular disease. ${ }^{7-9}$ Isoflavones act as selective oestrogen receptor (ER) modulators (SERMs), as ER agonists in low-oestrogen conditions and as ER antagonists in high-oestrogen conditions. ${ }^{10}$ Equol is a metabolite of a soy isoflavone, which is converted from daidzein by specific gut bacteria. ${ }^{11}$ Only $30 \%-60 \%$ of people can produce equol from daidzein after eating soy. ${ }^{12}$ Equol is more bioavailable than other soy isoflavones, and equol producers are believed to benefit from the consumption of soy isoflavones. ${ }^{13}$ It is plausible that 
isoflavones may stabilise the natural fluctuation of the oestrogen cycle through their SERM-like activities to alleviate PMS symptoms. ${ }^{14}$ A previous report demonstrated that soy isoflavone supplementation had beneficial effects for the relief of specific PMS symptoms such as cramp and swelling. ${ }^{15}$

\section{Equol production status and PMS}

Recently, we reported the relationship between PMS and equol production status and showed that equol non-production was a significant risk factor for PMS. ${ }^{16}$ Dietary interventions, such as supplementation with equol, might be a safe treatment strategy for PMS. A natural S-equol supplement (SE5-OH) was reported to be effective in treating menopause symptoms without any serious adverse effects. ${ }^{17}$

\section{TRIAL OBJECTIVE AND HYPOTHESIS}

The aim of this study is to examine the effect of natural SE5-OH on premenstrual symptoms. We hypothesise that equol is more effective than placebo in alleviating premenstrual symptoms in equol non-producers.

\section{METHODS AND ANALYSIS \\ Trial design}

This study is designed as a double-blind, placebo-controlled, parallel, randomised trial with a ratio of $1: 1$, in which a stratified randomisation is conducted with adjustment factors of the Daily Record of Severity of Problems
(DRSP) total score $(\geq 50$ or $<50)$ and age $(\geq 35$ or $<35$ years $)$ at baseline. The trial was registered at the University Hospital Medical Information Network (UMIN) centre (ID: UMIN000031815), and any important changes in the protocol will be reflected there. Table 1 shows the trial schedule.

\section{Setting and participants}

This trial will be conducted at the Research Institute of Traditional Asian Medicine, Kindai University Hospital. Recruitment will be by advertisement in local free papers and by posters displayed in Kindai University Hospital. The start of the recruitment is planned for May 2018, and it will be finished within 2 years.

Potential participants will receive oral and written information regarding the study by a doctor involved in this study.

\section{Eligibility criteria}

Eligible women for the trial must fulfil all of the following criteria:

- Age 20-45years.

- Presence of PMS symptoms and fulfil the 'moderate-to-severe PMS' or 'PMDD' criteria according to the Premenstrual Symptoms Questionnaire (PSQ). ${ }^{18}$

- Regular menstrual cycles (25-38 days).

- Non-equol producers (the equol-production status of the subjects will be determined by a soy challenge test, as described in the Study procedure section).

Table 1 Schedule of enrolment, interventions and assessments

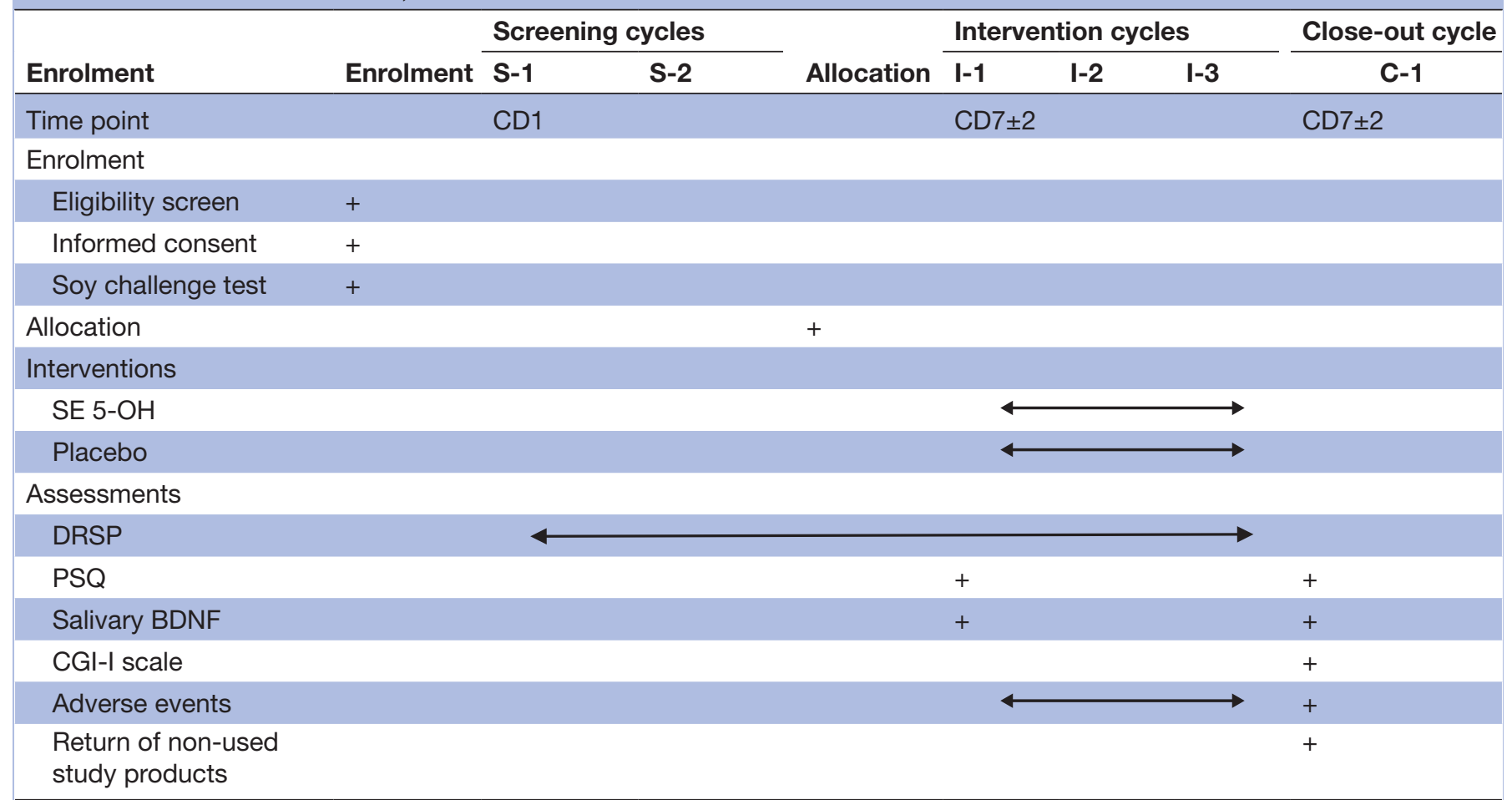

BDNF, brain-derived neurotrophic factor; CD, cycle day; CGI-I, Clinical Global Impressions-Improvement; DRSP, Daily Record of Severity of Problems; PSQ, Premenstrual Symptoms Questionnaire; SE5-OH, natural S-equol supplement. 
Subject recruitment and identification of eligibility

Excluded if ineligible

Qualification phase

Excluded if equol producers, and do not fulfill PMS criteria

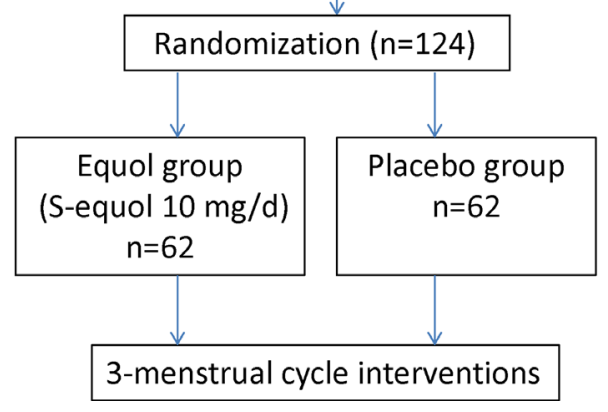

Figure 1 Flow chart of the study diagram.

- No oral conceptive pill use for 4 weeks before study entry.

- No concurrent therapy for PMS, including antidepressants, herbs and supplements, for 4 weeks before study entry.

- Provide written informed consent.

Study subjects will be excluded for the following reasons:

- Allergy to soybean or soybean products.

- Presence of neuropsychiatric disorders.

- Presence of very severe interference with work, usual activities or relationships by PMS symptoms.

- Serious hepatic disease, heart disease, kidney disease or malignant disease.

To meet the criteria for randomisation, study subjects need to fulfil two consecutive menstrual cycles of PMS criteria as measured by the DRSP ${ }^{19}$ immediately before the three double-blind placebo or active treatment cycles.

PMS severity criteria include the following:

- A postmenstrual phase (days 6-10) daily average $\leq 2.5$ for each symptom.

- A premenstrual phase (day -5 to -1 before menstruation) daily average $\geq 3$.0 for three distinct items.

- A premenstrual phase daily average worsened by at least $50 \%$ compared with the corresponding postmenstrual phase daily average for three distinct items.

- A score $\geq 3$ on functional impairment items for at least 1 day during the premenstrual phase.

\section{Interventions}

The intervention will be the administration of natural SE5-OH tablet containing $2.5 \mathrm{mg}$ S-equol produced from the fermentation of soy germ by Lactococcus 20-92. ${ }^{17}$ The placebo tablets will contain lactose in place of SE5-OH and the appearance will be the same as those of the active product. The study products (SE5-OH and placebo) will be manufactured as tablets and supplied by
Otsuka Pharmaceutical (Tokyo, Japan) free of charge. These tablets will be administered by a doctor involved in this study.

\section{Study procedure}

Figure 1 shows the study flow and table 1 shows the study time schedule. The study will include screening cycles (S-1 and S-2) and intervention cycles (I-1, I-2 and I-3). Subjects will receive oral and written information regarding the study. Written informed consent will be obtained by the physicians involved in the study.

\section{Screening cycles}

The equol-production status of the subjects will be determined by a soy challenge test. Participants will be instructed to eat soy food containing approximately $50 \mathrm{mg}$ isoflavones twice a day, and then first morning urine samples will be collected the next morning. These samples will be transported by mail to the Saga Nutraceuticals Research Institute of Otsuka Pharmaceutical. Then, the equol concentration will be measured by high-performance liquid chromatography using the modified method of Lundh et al..$^{20}$ The detection limit of equol in this assay is $0.85 \mathrm{nmol} / \mathrm{mL}$. Those subjects who have detectable urine equol will be classified as equol producers.

Only the non-equol producers will be selected and they will be required to check their premenstrual symptoms using the DRSP for two consecutive menstrual cycles. The DRSP comprises 21 items of emotional and physical PMS symptoms and 3 items of functional impairment for social and life activity, which are rated for their severity on a 6-point scale from 1 (not at all) to 6 (extreme). ${ }^{19}$

\section{Intervention cycles}

Eligible subjects will be randomly assigned to receive SE5-OH at a dose of $10 \mathrm{mg}$ S-equol or placebo, orally, twice daily, receiving four tablets $(2 \times 2$ tablets $)$ from day $7( \pm 2)$ onwards to the start of the next menstruation, during three menstruation cycles continuously. They will be required to check their PMS symptoms using the DRSP every day.

At the start and end of the intervention, on day $7( \pm 2)$ of the I-1 and C-1 cycles, subjects will be required to collect their saliva samples themselves using a Saliva Collection Aid (Salimetrics, Carlsbad, California, USA). At that time, they will also be required to check their PMS symptoms using the PSQ.

After the intervention, they will be checked at a final assessment during the close-up cycle (C-1). The Clinical Global Impressions-Improvement (CGI-I) scale will be checked by a physician, and then the involvement of the study subjects in the trial will cease.

\section{Outcome measure}

The primary outcome measure consists of 21 symptom items of the DRSP. The average of the premenstrual phase (days -5 to -1 before menstruation) for 21 items will be summed to generate the DRSP total score. The primary efficacy variable is the difference between the DRSP total 
score from the screening cycle (S-2) and the DRSP total score from the last intervention cycle (I-3).

The secondary outcome measurements are the percentage difference of the DRSP total score between S-2 and I-3, the difference between the salivary brain-derived neurotrophic factor (BDNF) from baseline to the end of treatment, and the ratio of mild PMS in the PSQ and CGI-I scale at the end of treatment.

Saliva will be stored at $-20^{\circ} \mathrm{C}$ until assayed. The BDNF will be detected by sandwich ELISA according to the manufacturer's instructions (CYT306; Millipore, Bedford, Massachusetts, USA) as described previously. ${ }^{21}$ All samples will be tested in duplicate and the mean value will be determined.

The CGI-I scale is a single-item, 7-point scale measure ( $1=$ very much improved to $7=$ very much worse). We will define the response group as having a score of 1 or 2, corresponding to very much improved or much improved.

\section{Sample size calculation}

A pilot study of 10 participants was conducted to estimate the sample size. The result of this open-label pilot study showed that the change from baseline in the mean $( \pm$ SD) DRSP total score of SE5-OH treatment was $-14.2( \pm 15.4)$. As this pilot study included low DRSP total score cases, we expect that SE5-OH will achieve much better improvement of the DRSP total score in this study. According to a previous report studying the effect of calcium for the treatment of PMS by doubleblind, randomised, placebo-controlled trial, ${ }^{22}$ the mean change in the DRSP total score of placebo treatment from baseline was -7.6 . Accordingly, we assumed that the SE5-OH and placebo groups will show a mean decrease in DRSP total score from baseline of 16 and 8 , respectively, with an SD of 15 for each group. Under these assumptions, the required sample size was calculated as 114 ( 57 in each group) with a significance level of $5 \%$ (two-sided) and power of $80 \%$, where the Student's t-test was applied. Accounting for the dropout of a few subjects, we set the target sample size as 124 (62 in each group).

\section{Randomisation}

A randomisation list will be generated using a computer program by the Data Coordinating Center of Osaka University Hospital (Osaka, Japan). This procedure will be performed by a physician not involved in the study.

\section{Blinding}

The active product and placebo will be packaged in identical bottles and sealed. These are similar in look and taste. All participants and investigators will be blinded to the assigned treatment throughout the study.

\section{Data collection and management}

All subjects will be assigned a study identification number. Case report forms (CRFs) will be in paper form. Data will be entered and stored in an electronic database, which is managed by the Data Coordinating Center of Osaka
University Hospital. All CRFs and study data will be stored in a locker within Kindai University.

\section{Monitoring}

An independent Data and Safety Monitoring Board (DSMB) will be set up prior to the start of the study. The DSMB will review data after the first participant, and then after the recruitment of $25 \%, 50 \%$ and $75 \%$ of participants to check the study progress and all adverse events.

\section{Statistical analysis}

All efficacy variables will be analysed for the full analysis set, which is defined as all randomised subjects who have at least 1 day DRSP total score measurement at S-2 and I-3, without major protocol violations. The safety analysis set will consist of subjects who receive the study tablets at least once and who undergo safety assessment.

Statistical analysis will be performed using the SAS V.9.4 statistical software.

Baseline characteristics will be summarised for each group. Continuous variables will be presented using mean, median, SD and range. Categorical variables will be presented as percentage.

To compare the primary outcomes (difference of DRSP total score between S-2 and I-3) between the SE5-OH group and the placebo group, the difference between the mean differences in these two groups will be determined with $95 \%$ CI. Furthermore, the p value will be determined using Student's t-test, where the significance level is 5\% (two-sided). The secondary outcomes (percentage difference of DRSP total score and salivary BDNF) will also be compared using the same summary measures. PSQ and CGI-I will be summarised as the difference of effective proportions with $95 \% \mathrm{CI}$, where $\mathrm{p}$ value will be determined using Fisher's exact test.

\section{Safety assessment}

All subjects will be required to report any adverse event during the trial. All serious adverse events will be immediately reported to the project leader, ethics committee, all participating investigators and the manufacturer of the study products.

\section{Patient and public involvement}

The research question on the effect of natural SE5-OH on premenstrual symptoms was first proposed by a patient who failed the standard treatment. Patients were not involved in the planning of this study. The results will be disseminated to study participants via the website of our research institute.

\section{ETHICS AND DISSEMINATION}

Any modification in the protocol will be presented to the ethics committee. The results will be submitted to a peer-reviewed journal. Abstracts will be submitted to relevant national and international conferences. This 
study will follow the standards set by the guidelines of the Consolidated Standards of Reporting Trials.

Acknowledgements We thank the Edanz Group (www.edanzediting.com/ac) for editing a draft of this manuscript.

Contributors TT, MS and YC contributed to the drafting of the manuscript. TT was the main contributor to the study design and conception. All authors agreed with the integrity of the study and gave their approval.

Funding This work was supported, in part, by a grant from Research, AMED Research Project for Improving Quality in Healthcare and Collecting Scientific Evidence on Integrative Medicine (grant number 17933369), Tokyo, Japan.

Competing interests $T T$ received lecture fees from Otsuka Pharmaceutical, the manufacturer of SE5-OH. Otsuka Pharmaceutical will have no role in the conception, study planning, data analysis or interpretation of the data.

Patient consent Not required.

Ethics approval The trial protocol has been approved by the Ethics Committee of Kindai University (approval number 29-202).

Provenance and peer review Not commissioned; externally peer reviewed.

Open access This is an open access article distributed in accordance with the Creative Commons Attribution Non Commercial (CC BY-NC 4.0) license, which permits others to distribute, remix, adapt, build upon this work non-commercially, and license their derivative works on different terms, provided the original work is properly cited, appropriate credit is given, any changes made indicated, and the use is non-commercial. See: http://creativecommons.org/licenses/by-nc/4.0/.

\section{REFERENCES}

1. Yonkers KA, O'Brien PM, Eriksson E. Premenstrual syndrome. Lancet 2008;371:1200-10.

2. Angst J, Sellaro R, Merikangas KR, et al. The epidemiology of perimenstrual psychological symptoms. Acta Psychiatr Scand 2001;104:110-6.

3. Dimmock PW, Wyatt KM, Jones PW, et al. Efficacy of selective serotonin-reuptake inhibitors in premenstrual syndrome: a systematic review. Lancet 2000;356:1131-6.

4. Association AP. Diagnostic and statistical manual of mental disorders: DSM-5. Arlington: American Psychiatric Association 2013.

5. Grady-Weliky TA. Clinical practice. Premenstrual dysphoric disorder. N Engl J Med 2003;348:433-8.

6. Kelderhouse K, Taylor JS. A review of treatment and management modalities for premenstrual dysphoric disorder. Nurs Womens Health 2013;17:294-305.
7. Setchell KD. Phytoestrogens: the biochemistry, physiology, and implications for human health of soy isoflavones. Am J Clin Nutr 1998;68-1333S-46.

8. Cornwell T, Cohick W, Raskin I. Dietary phytoestrogens and health. Phytochemistry 2004;65:995-1016.

9. Cassidy A, Albertazzi P, Lise Nielsen I, Nielsen L I, et al. Critical review of health effects of soyabean phyto-oestrogens in postmenopausal women. Proc Nutr Soc 2006;65:76-92.

10. Izumi T, Piskula MK, Osawa S, et al. Soy isoflavone aglycones are absorbed faster and in higher amounts than their glucosides in humans. J Nutr 2000;130:1695-9.

11. Atkinson $\mathrm{C}$, Berman $\mathrm{S}$, Humbert $\mathrm{O}$, et al. In vitro incubation of human feces with daidzein and antibiotics suggests interindividual differences in the bacteria responsible for equol production. J Nutr 2004;134:596-9.

12. Atkinson C, Frankenfeld CL, Lampe JW. Gut bacterial metabolism of the soy isoflavone daidzein: exploring the relevance to human health. Experimental biology and medicine (Maywood, N. J.) 2005;230:155-70.

13. Shor D, Sathyapalan T, Atkin SL, et al. Does equol production determine soy endocrine effects? Eur J Nutr 2012;51:389-98.

14. Kurzer MS. Hormonal effects of soy in premenopausal women and men. J Nutr 2002;132-570S-3.

15. Bryant M, Cassidy A, Hill C, et al. Effect of consumption of soy isoflavones on behavioural, somatic and affective symptoms in women with premenstrual syndrome. Br J Nutr 2005;93:731.

16. Takeda T, Ueno T, Uchiyama S, et al. Relation between premenstrual syndrome and equol-production status. J Obstet Gynaecol Res 2016;42:1575-80.

17. Aso T, Uchiyama S, Matsumura Y, et al. A natural S-equol supplement alleviates hot flushes and other menopausal symptoms in equol nonproducing postmenopausal Japanese women. $J$ Womens Health2012;21:92-100.

18. Takeda T, Tasaka K, Sakata M, et al. Prevalence of premenstrual syndrome and premenstrual dysphoric disorder in Japanese women. Arch Womens Ment Health 2006;9:209-12.

19. Endicott J, Nee J, Harrison W. Daily Record of Severity of Problems (DRSP): reliability and validity. Arch Womens Ment Health 2006;9:41-9.

20. Lundh TJ, Pettersson H, Kiessling KH. Liquid chromatographic determination of the estrogens daidzein, formononetin, coumestrol, and equol in bovine blood plasma and urine. J Assoc Off Anal Chem 1988;71:938-41.

21. Matsuki C, To M, Kondo Y, et al. Associations between brainderived neurotrophic factor and estradiol in women's saliva. Neuro Endocrinol Lett 2014;35:236-41.

22. Shehata NA. Calcium versus oral contraceptive pills containing drospirenone for the treatment of mild to moderate premenstrual syndrome: a double blind randomized placebo controlled trial. Eur J Obstet Gynecol Reprod Biol 2016;198:100-4. 